\title{
EMOTIONAL IMPACT IN FRONTLINE AND SECONDLINE HEALTHCARE PROFESSIONALS: COVID-19 AND II WAVE
}

\author{
Federica Guerra $^{1}$, Jessica Ranieri ${ }^{1}$, Claudio Ferri ${ }^{1,2}$, \& Dina Di Giacomo ${ }^{1}$ \\ ${ }^{I}$ Department of life, Health and Environmental Sciences, University of L'Aquila (Italy) \\ ${ }^{2}$ Internal Medicine Division, S. Salvatore Hospital, ASL 1 AQ (Iyaly)
}

\begin{abstract}
Introduction. The rapid spread of coronavirus disease 2019 (COVID-19) has created unprecedent global challenges for health systems. National Healthcare Systems Hospitals adopted protective measures and medical equipment resources, exposing healthcare workers at risk for stress syndromes, subclinical mental health symptoms, and long-term occupational burnout. Health workers have had to deal with the most severe clinical cases in intensive care specialized operative division. Since the first months of the epidemic spread, some studies have established shown that nurses have shown symptoms of severe anxiety associated with peritraumatic dissociative experiences. Most of the studies examined the emotional impact of COVID 19 on health professionals but did not focus on different consider professionals roles and hospital departments workload.

Objective. The aim of our study was to analyze the emotional characteristics of health workers during the II wave of coronavirus (November-December 2020), comparing the frontline (COVID 19) and second line (chronic diseases) hospital divisions and analyzing the differences between the health roles.

Methods. We conduct a pilot study among health-workers. A sample of 28 healthcare workers (aged 23-62 years) were recruited from frontline and secondline hospital departments (L'Aquila, IT). The administered psychological battery was composed of n. 4 self-reports evaluating emotional variables (depression, anxiety, and stress) (DASS-21), personality traits (BFI-10), burnout risk (MBI), and perceived stress (PSS).

Results. The results highlighted significant differences: older health workers were found to be more vulnerable than those who younger health workers; another interesting point was that healthcare workers serving in frontline wards showed symptoms of depersonalization. No significant difference for the type of role held.

Conclusions. A prevention program should be activated to preserve frontline and older workers mental health. Earlier support could mitigate the effect of the pandemic experience, reducing the risk for emotional health workers' fragility.
\end{abstract}

Keywords: Covid-19, healthcare workers, emotional impact, clinical psychology, mental health.

\section{Introduction}

The covid-19 pandemic has put healthcare workers around the world in an unprecedented situation. Healthcare professionals have had to balance their physical and mental health care needs with those of critically ill patients with limited or inadequate resources and align their desire and duty to patients with those to family and friends. This could create some moral injuries or mental health problems. Hospitals have had to put protective measures and medical equipment resources in place, exposing health care workers to the risk of stress syndromes, mental health symptoms subclinical and long-term professional burnout; depression, anxiety, peritraumatic dissociative experience and mental illness were detected as a response in acute COVID-19. Healthcare workers at greater risk of exposure to the virus suffer a greater psychological impact than those with less exposure. Ranieri et al., conducted a study on female nurses during the pandemic, highlighting anxiety and post-traumatic stress disorder; individual resources including personality dimensions did not mediate emotional distress or the likely risk of PTSD: the impact of the pandemic on the mental health of nurses was strong and unmanageable by itself. In China, frontline health workers who directly care for COVID-19 patients experienced stress, anxiety and insomnia and showed higher levels of severe mental health symptoms than those in secondary roles. Most of the studies looked at the emotional impact of COVID 19 on healthcare workers but did not focus on different roles of health-workers and hospital ward workload. 


\section{Study design}

Participants have been enrolled in Hospital of L'Aquila (Italy). Informed consent was obtained from each participant at the time of enrolment and the study adhered to the Declaration of Helsinki.

A pilot study was conducted among healthcare workers, working in internal medicine and nephrology, oncology, and general and transplant surgery at the hospital, during the second wave of Coronavirus (November and December 2020). Trained clinical psychologists, blind to the objectives of the study, conducted the psychological screening in a quiet, dedicated room. The duration of the evaluations was 15 minutes. Data were collected anonymously.

\section{Objectives}

The aim of our study was to investigate the emotional conditions of healthcare workers (OSS, nurses, and doctors) during the II wave of COVID-19 and the role of personality characteristics in coping with stressful situations.

\section{Methods}

\section{Ethical Approval}

This study was approved by the Internal Review Board (IRB) of the University of L'Aquila, Italy (Prot. No. 31457/2020). Written informed consent was obtained following the Helsinki Declaration (WMA).

\subsection{Participants}

Eligible participants were 28 healthcare workers (nurses, $n=10$; doctor, $n=12$; oss, $n=6$ ), aged 23-62 years old (mean age 39,8; sd \pm 12 ). Participants were recruited in three worked unit: internal medicine and nephrology (named frontline) (n 11; 39,2\%), oncology (named secondline) (n 6, 21,4\%) and general and transplant surgery (named secondline) (n 11, 39,2\%). Further, 23 participants were women $(82,1 \%)$, and 5 participants were men $(17,8 \%)$.

\subsection{Measurement}

The characteristic of the participant, age, relationship status and qualification were assessed using a socio-demographic form. We also assessed personality traits, depression, anxiety, and stress symptoms, peritraumatic dissociation, perceived stress, and burnout. Psychological battery has been composed of n.4 self-reports evaluating the depression, anxiety, and stress (DASS-21), personality traits (BFI-10), burnout (MBI) and perceived stress (PSS). Big Five Inventory-10 (BFI-10). The BFI-10 (Guido et al., 2015) evaluates the five personality dimensions on a 5-point scale ranging from 1 (strongly disagree) to 5 (strongly agree), each with two items: openness (OP), conscientiousness (CO), emotional stability (ES), extraversion (EX), and agreeableness (AG). Depression, Anxiety and Stress Scale (DASS-21) (Bottesi et al., 2017). The DASS is a clinical assessment that measures the three related states of depression, anxiety, and stress. It has 21 questions and takes about 3 minutes to complete. Each subscale measuring the emotional traits is composed of 7 items. Peritraumatic Dissociative Experiences Questionnaire (PDEQ) (Weiss et al, 2017). It is a 10-items self-report questionnaire measuring peritraumatic dissociation. The PDEQ has well-established psychometric properties, with higher total scores indicating increased peritraumatic dissociation. A score above 15 is indicative of significant dissociation. Maslach Burnout Inventory (MBI) (Maslach C. et al., 1981). The MBI is a 22 items self-report questionnaire measuring the incidence of burnout and of its three domains: emotional exhaustion, depersonalization and reduced professional achievement. Perceived Stress Scale (PSS) (Cohen S., 1988). It is a 10-items self-report questionnaire measuring perceived stress on a 4-point scale ranging from 0 (never) to 4 (very often).

\section{Statistical analysis}

Descriptive statistics and analysis of variance (ANOVA) test as appropriate, we compared emotional severity by demographic variables during the second wave of the COVID-19 in Italy. Spearman rank order correlation was used to examine correlations among depression, anxiety, stress, peritraumatic stress, perceived stress, burnout, and personality traits. All statistical analyses were conducted using Jamovi and the $\alpha$-value was set as $\mathrm{p} \leq 0.05$. 


\section{Results}

Comparing total psychological test scores by age group (median $=40$ ) and by department (frontline and secondline), the data showed signs of burnout and psychological distress particularly in older health workers working on the frontline (see Table 1).

Table 1. Raw scores of the sample groups on psychological assessment.

\begin{tabular}{|c|c|c|c|c|c|c|c|}
\hline \multirow[t]{3}{*}{ Tests/Groups } & \multicolumn{3}{|c|}{ Younger } & \multicolumn{4}{|c|}{ Older } \\
\hline & $\begin{array}{l}\text { Frontline } \\
\text { (n 7) }\end{array}$ & \multicolumn{2}{|c|}{$\begin{array}{l}\text { Secondline } \\
\text { (n 7) }\end{array}$} & \multicolumn{2}{|c|}{$\begin{array}{c}\text { Frontline } \\
\text { (n 4) }\end{array}$} & \multicolumn{2}{|c|}{$\begin{array}{l}\text { Secondline } \\
\text { (n 10) }\end{array}$} \\
\hline & SD & $\mathrm{X}$ & SD & $\mathrm{X}$ & SD & $\mathrm{X}$ & SD \\
\hline PSS & $12.9 \pm 6.82$ & \multicolumn{2}{|c|}{ 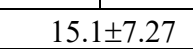 } & \multicolumn{2}{|c|}{$\frac{1}{17.3 \pm 4.50}$} & \multicolumn{2}{|c|}{$22.5 \pm 7.38$} \\
\hline $\begin{array}{l}\text { Ee } \\
\text { De } \\
\text { Re }\end{array}$ & $\begin{array}{l}8.57 \pm 4.08 \\
2.71 \pm 2.36 \\
40.3 \pm 7.04\end{array}$ & \multicolumn{2}{|c|}{$\begin{array}{l}12.9 \pm 7.86 \\
3.57 \pm 4.76 \\
41.4 \pm 5.00\end{array}$} & \multicolumn{2}{|c|}{$\begin{array}{l}22.8 \pm 12.2 \\
12.3 \pm 7.63 \\
39.0 \pm 5.48\end{array}$} & \multicolumn{2}{|c|}{$\begin{array}{l}14.7 \pm 7.35 \\
3.80 \pm 6.61 \\
36.6 \pm 6.33\end{array}$} \\
\hline DASS-21 & $\begin{array}{r}3.71 \pm 5.35 \\
2.86 \pm 3.63 \\
13.4 \pm 5.86\end{array}$ & \multicolumn{2}{|c|}{$\begin{array}{l}10.3 \pm 9.55 \\
12.9 \pm 11.2 \\
12.0 \pm 7.75\end{array}$} & \multicolumn{2}{|c|}{$\begin{array}{l}15.0 \pm 10.6 \\
9.00 \pm 6.83 \\
15.0 \pm 10.9\end{array}$} & \multicolumn{2}{|c|}{$\begin{array}{l}9.80 \pm 8.56 \\
5.00 \pm 4.45 \\
15.6 \pm 7.76\end{array}$} \\
\hline $\begin{array}{cr}\text { BFI-10 } & \\
& \text { Agr } \\
\text { Con } \\
\text { Em. Stab } \\
\text { Ext } \\
\text { Ope }\end{array}$ & $\begin{array}{r}5.71 \pm 1.70 \\
8.14 \pm 1.68 \\
6.00 \pm 1.41 \\
6.14 \pm 1.95 \\
8.14 \pm 1.68\end{array}$ & \multicolumn{2}{|c|}{$\begin{array}{l}7.14 \pm 1.21 \\
9 \pm 1.91 \\
7 \pm 1.63 \\
\quad 6.29 \pm 2.69 \\
6.86 \pm 1.68\end{array}$} & \multicolumn{2}{|c|}{$\begin{array}{r}4.50 \pm 1.29 \\
9.25 \pm 1.50 \\
7.00 \pm 1.41 \\
7.50 \pm 2.08 \\
6.75 \pm 1.71\end{array}$} & \multicolumn{2}{|c|}{$\begin{array}{r}5.10 \pm 1.52 \\
8.20 \pm 1.87 \\
6.20 \pm 1.69 \\
6.10 \pm 1.97 \\
5.60 \pm 2.17\end{array}$} \\
\hline
\end{tabular}

First, we compared (Kruskal-Wallis One-way ANOVA non-parametric) demographic variables with emotional variables. The analysis revealed that there was a significant difference between age group. The older healthcare worker showed greater perceived stress (PSS) than the younger group $(\mathrm{p}=0.014)$ (Figure 1).

Figure 1. Representation of perceived stress (PSS) score by Age group.

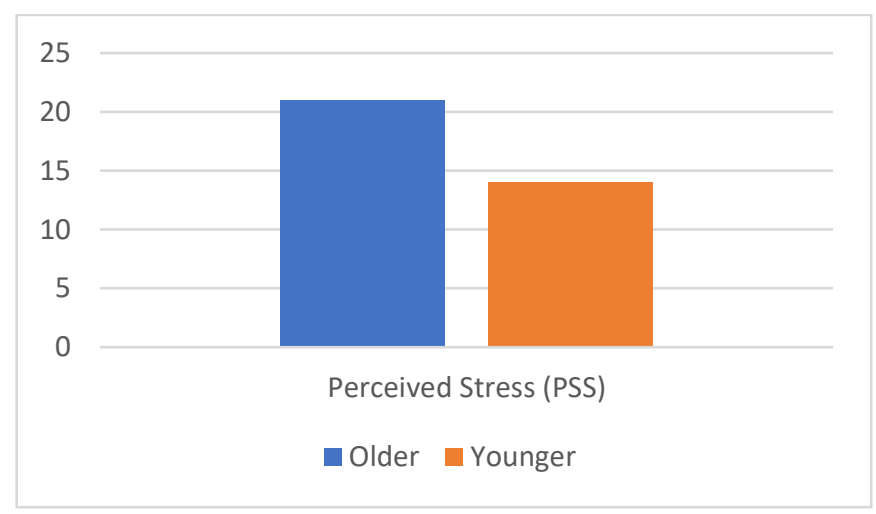

No significant difference between worked, qualification (doctor, nurse, OSS) or demographic characteristic (gender, marital status) and the other emotional variables (DASS-21 and MBI).

Correlation analysis (Spearman test) was performed among BFI-10, DASS-21, PSS and MBI. We examined the correlation between burnout level and emotional indices. Spearmen's correlation analysis revealed that there was a negative relationship between scores on the reduced professional achievement (MBI) and perceived stress (PSS) $(\mathrm{r}=-0.43 ; \mathrm{p}=0.022)$. Furthermore, positive correlation was highlighted between MBI and DASS-21 and then PSS. In particular, depression (DASS-21) shows positive correlations with emotional exhaustion (MBI) $(\mathrm{r}=0.497 ; \mathrm{p}=0.007)$ and perceived stress (PSS) $(\mathrm{r}=0.600 ; \mathrm{p}=0.001)$; anxiety (DASS-21) shows positive correlations with emotional exhaustion (MBI) $(r=0.502 ; p=0.006)$, 
depersonalization $(\mathrm{r}=0.446 ; \mathrm{p}=0.017)$ and depression $(\mathrm{r}=0.548 ; \mathrm{p}=0.003)$; stress (DASS-21) shows positive correlations with perceived stress $(\mathrm{r}=0.561 ; \mathrm{p}=0.002)$, depression $(\mathrm{r}=0.683 ; \mathrm{p}=0.001)$ and anxiety $(\mathrm{r}=0.578$; $\mathrm{p}=0.001$ ).

Afterward, we wanted to explain the relation between emotional traits and BFI-10 indexes. Agreeableness, extroversion, and openness showed correlation with MBI and PSS. In particular, agreeableness showed negative correlation with emotional exhaustion (MBI) $(r=-0.516 ; p=0.005)$, depersonalization $(\mathrm{r}=-0.540 ; \mathrm{p}=0.003)$, and perceived stress (PSS) $(\mathrm{r}=-0.412 ; \mathrm{p}=0.029)$; extroversion showed negative correlation with perceived stress (PSS) $(\mathrm{r}=-0.408 ; \mathrm{p}=0.031)$; openness showed negative correlation with perceived stress (PSS) $(\mathrm{r}=-0.403 ; \mathrm{p}=0.033)$ and positive correlation with reduced professional achievement (MBI) $(\mathrm{r}=0.491 ; \mathrm{p}=0.008)$. There was no significant difference in conscientiousness and emotional stability.

\section{Discussion and conclusion}

This pilot study was focused on the screening of mental health in healthcare worker during $2^{\text {nd }}$ wave of COVID-19; we wanted to investigate the role of the personality traits such as a protective psychological factor. Age and type of hospital ward influenced the emotional impact and level of burnout in the healthcare professions.

Faced with the second wave of the COVID-19 pandemic, healthcare workers showed in the short time the risk of developing emotional exhaustion and depersonalization (from high risk to low risk) and perceived stress related to depression, anxiety, and stress. Elderly and healthcare professionals working in the frontline unit showed a higher risk of developing emotional distress and burnout. This outcome may be attributable to the greater exposure with COVID-19 patients compared to the other two departments that dealing with chronic diseases (secondline) and a social factor such as having a family and this could explain the greater discomfort of older health workers for fear of infecting loved ones (e.g children, husband). Indeed, among the mental health risk factors for healthcare professionals, a study identified mental exhaustion, anxiety about being infected and infecting the family.

The dimensions of the personality related to the emotional variables were the agreeableness (based on positive feeling, sincere and trusting), the extroversion (based on sociability, friendliness and energy) and the openness (open to experience, intellectually curios, open to emotions, sensitive to beauty).

This study highlights that age and personality traits may mediate the likely risk of burnout symptoms (in particular, emotional exhaustion and depersonalization) and perceived stress: Young health care workers appeared with increased emotional well-being. Their high level of agreeableness helps them overcome the impact of the public emergency. Conversely, a high level of openness predicts a high risk of burnout syndrome during development

Our study showed that personality traits can influence the psychological wellness during emergency.

The present findings suggest the need for regular screening of psychological distress even in healthcare workers, paying to attention to the personality traits, age, and hospital ward, especially in emergency situations such as the COVID-19 pandemic.

\section{References}

Blake H., Bermingham F., Johnson G., Tabner A. (2020). Mitigating the Psychological Impact of COVID-19 on Healthcare Workers: A Digital Learning Package. Int. J. Environ. Res. Public Health, 17, 9 .

Bottesi G., Ghisi M., Altoè G., Conforti E., Melli G., Sica C., (2015) The Italian version of the Depression Anxiety Stress Scales-21: Factor structure and psychometric properties on community and clinical samples. Comprehensive Psychiatry 60, 170-181

Cohen, S. and Williamson, G. (1988). Perceived Stress in a Probability Sample of the United States. The Social Psychology of Health. Newbury Park

Greenberg N, Docherty M, Gnanapragasam S, Wessely S. (2020). Managing mental health challenges faced by healthcare workers during covid-19 pandemic. $B M J, 368$.

Guido G., Peluso A.M., Capestro M., Miglietta M. (2015). An Italian version of the 10-item Big Five Inventory: An application to hedonic and utilitarian shopping values Personality and Individual Differences 76,135-140

Marmar C., Metzler J. T., Otte C., McCaslin S., Inslicht S.S., Haase H.C. (2007). The Peritraumatic Dissociative Experiences Questionnaire an International Perspective. Cross-Cultural Assessment of Psychological Trauma and PTSD, 197-217 
Maslach C., Jackson E., S. (1981). The measurement of experienced burnout. Journal of Organization Behavior, 2, 99-113

Mosheva M., Gross R., Palmor H.M., Ohayon H.I., Kaplan R., Cleper R., Kreiss Y., Gothelf D., Pessach M.I. (2021). The association between witnessing patient death and mental health outcomes in frontline COVID-19 healthcare workers. $A D A A$

Ranieri J., Guerra F., Di Giacomo D. (2020). Predictive risk factors for post-traumatic stress symptoms among nurses during the Italian acute COVID-19 outbreak. Health Psychology Report, 8,1.

Robles R., Palacios M., Rangel N., Real T., Becerra B., Fresán A., Vega H., Rodríguez E., Durand S., Madrigal E., (2020). A qualitative assessment of psycho-educational videos for frontline COVID-19 healthcare workers in Mexico. ISSN, Vol. 43, 6 